\title{
Experimental study on the characteristics of mixed molding fuel with sludge and corn stalk
}

\author{
Wen-Yang Sun, Li-Ming Wang*, Yan-Ru Ma, \\ Yang Lv, Kang Feng and Wen-Tao Li \\ College of Engineering, Heilongjiang Bayi Agricultural University \\ Daqing, China \\ E-mail:870548757@qq.com
}

\begin{abstract}
To solve the problem of utilization of typical waste resources in Daqing, the rich corn stalk in Daqing and the Daqing oilfield waste sludge were respectively selected as major and auxiliary raw. The influence of sludge proportion, moisture content of mixed materials and molding pressure on quality characteristics of mixed molding was investigated. Maximum pressure and deformation displacement were introduced to measure the mechanical properties of molding fuel. The results showed that the sludge proportion was from $5 \%$ to $10 \%$, the moisture content of mixed materials was from $10 \%$ to $12 \%$, and the molding pressure was from $25 \mathrm{Mpa}$ to $30 \mathrm{Mpa}$. Under this condition, physical qualities of molding fuel were better. This study may provide a theoretical basis for making molding fuel with mixed materials and providing a new consideration for the resource utilization of sludge.
\end{abstract}

Keywords: Molding Fuel; Sludge; Corn Stalk; Physical Quality

\section{Introduction}

Facing with the situation that our country is more dependent on coal resources and has less energy reserves and low production, while energy demand is in a substantial increase, combining the present situation that our country is rich in straw resources in agriculture and the amount of straw resources available to collect is 687 million tons, but the abandoned and burned part of the straw is about $31.31 \%$, rational use of resources is particularly important. At present, molding fuel is one of the efficient and clean utilization of straw resources. Single material is mainly used to prepare traditional molding fuel. The study of molding fuel with mixed materials is limited to the introduction of urban sewage sludge in biomass materials or the introduction of biomass materials in briquette.

Combining resources advantages in Daqing, waste sludge in Daqing oilfield that generates in the oil exploration, mining, transportation, storage and refining process was added to the feedstock in this experiment. Sludge and corn 
stalk were mixed to produce molding fuel. Quality characteristics of molding fuel were observed under the condition of different sludge proportion, different moisture content of mixed materials and different molding pressure.

\section{Materials and Methods}

\subsection{Test materials and equipments}

Sludge and corn stalk were selected as raw materials. The main instruments and equipments are shown in Table 1 and the self-made three-dimensional compression device is shown in Figure 1.

Table 1. Instruments and equipments

\begin{tabular}{ll}
\hline The names of instruments and equipments & Model \\
\hline Hydraulic molding machine & YJ-1000 \\
Grinder & SG40 \\
Isotherm automatic calorimeter & $5 E-A C 8018$ \\
Moisture analyzer & MB25 \\
Computer-controlled electronic universal testing machine & WDW3100 \\
\hline
\end{tabular}

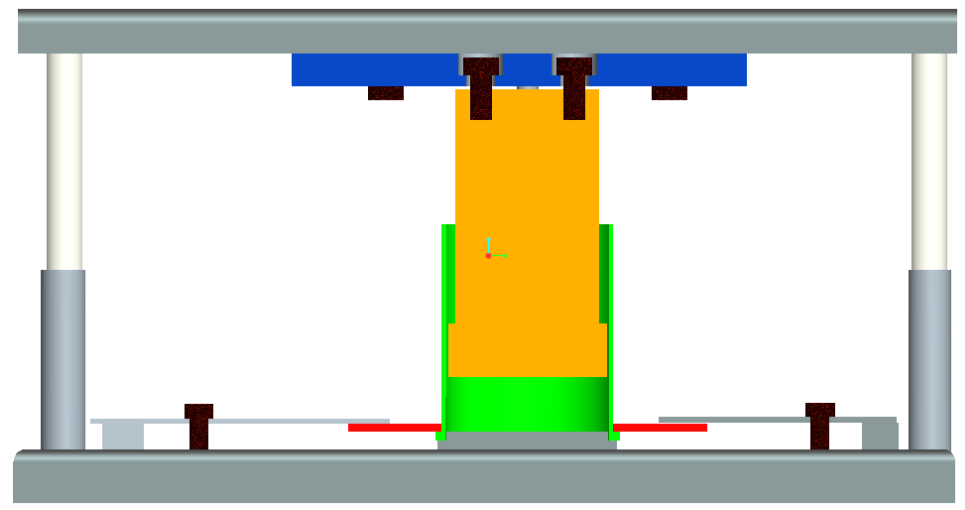

Fig. 1. The diagram of self-made three-dimensional compression device

\subsection{Test method}

(a) Calorific of molding fuel

According to GB / T213-2003 "Determination of calorific value of coal", the calorific of molding fuel under different sludge proportion was measured. Two samples were taken in this test.

(b) Moisture content of mixed materials

Measurement method was as follow: according to moisture test procedure 
of moisture analyzer, the heating temperature and heating time were sited. The test samples were prepared basing on the requirements of the test, and then heating mantle was closed. The test button was put on to start the test. After the test, the results were recorded.

(c) Deformation resistance

Under continuous loading until $30 \mathrm{kN}$ displacement of molding fuel were measured and maximum pressure under continuous loading deformation rupture were measured.

\section{Results and Analysis}

\subsection{Effects of sludge proportion on quality of molding fuel}

The calorific of molding fuel under different sludge proportions was shown in Figure 2. As sludge proportion increased, the calorific of molding fuel increased. The force and displacement diagram of molding fuel of different sludge proportions under continuous loading until 30kN was shown in Figure 3(a). As sludge proportion increased, the deformation displacement of molding fuel increased, and then tended to gently. When sludge proportion was from $5 \%$ to $10 \%$, under certain conditions of the force, the deformation displacement of molding fuel was smaller. This showed that deformation resistance of molding fuel was better. The curve of force changing with time of molding fuel of different sludge proportions under continuous loading was shown in Figure 3(b). When sludge proportion was over $10 \%$, the maximum pressure of deformation rupture of molding fuel was lower. Therefore, considering the calorific of molding fuel and deformation resistance, sludge proportion was chose from $5 \%$ to $10 \%$.

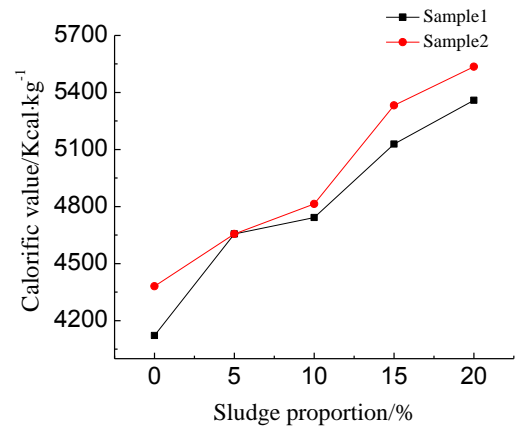

Fig. 2. The diagram of the relationship between sludge proportion and calorific of molding fuel 


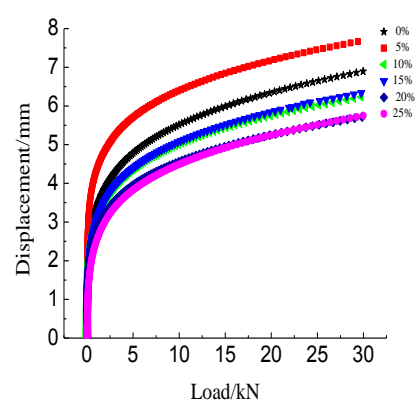

(a)

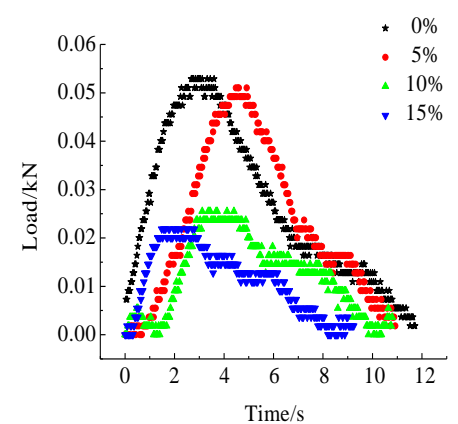

(b)

Fig. 3. The diagram of the relationship between sludge proportion and mechanical properties of the physical quality of molding fuel

\subsection{Effects of moisture content of mixed materials on the physical quality of molding fuel}

The force and displacement diagram of molding fuel of different moisture content of mixed materials under continuous loading until $30 \mathrm{kN}$ was shown in Figure 4(a). When moisture content of mixed materials was from $10 \%$ to $12 \%$, under certain conditions of the force, the deformation displacement of molding fuel was smaller. At this time, deformation resistance of molding fuel was better. The curve of force changing with time of molding fuel of different moisture content of mixed materials under continuous loading was shown in Figure 4(b). As moisture content of mixed materials increased, the maximum pressure of deformation rupture of molding fuel decreased slightly. Therefore, considering deformation displacement and maximum pressure of molding fuel, moisture content of mixed materials was chose from $10 \%$ to $12 \%$.

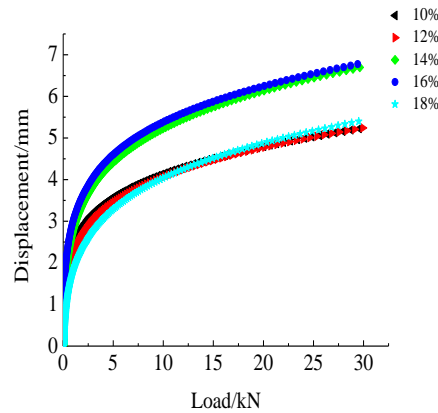

(a)

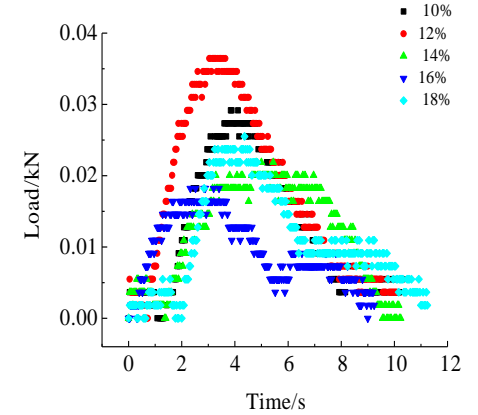

(b)

Fig. 4. The diagram of the relationship between moisture content of mixed materials and mechanical properties of the physical quality of molding fuel 


\subsection{Effects of molding pressure on the physical quality of molding fuel}

The force and displacement diagram of molding fuel of different molding pressure under continuous loading until 30kN was shown in Figure 5(a). As molding pressure increased, the deformation displacement of molding fuel increased, and then tended to gently. Under certain conditions of the force, when molding pressure was $30 \mathrm{Mpa}$, the deformation displacement of molding fuel was smallest. The curve of force changing with time of molding fuel of different molding pressure under continuous loading was shown in Figure 5(b). When molding pressure was from $25 \mathrm{mpa}$ to $30 \mathrm{mpa}$, the maximum pressure of deformation rupture of molding fuel was larger. Therefore, molding pressure was chose from $25 \mathrm{mpa}$ to $30 \mathrm{mpa}$, at this time, the physical quality of molding fuel was better.

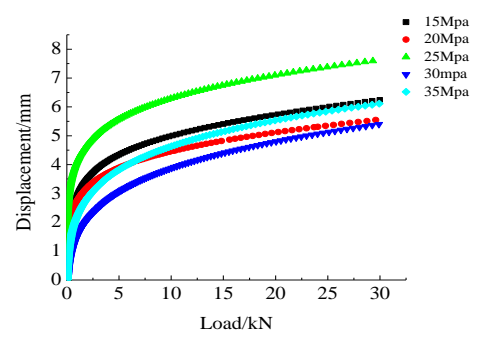

(a)

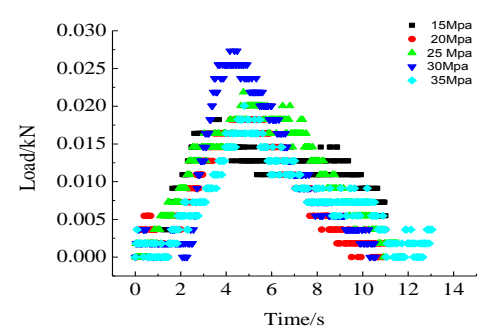

Fig. 5. The diagram of the relationship between molding pressure and mechanical properties of the physical quality of molding fuel

\section{Conclusion}

Maximum pressure and deformation displacement were introduced to measure the mechanical properties of molding fuel. The influence of sludge proportion, moisture content of mixed materials and molding pressure on quality characteristics of mixed molding was studied. The results were as follows:

(a) When sludge proportion was from $5 \%$ to $10 \%$, the combustion and physical properties of molding fuel were better.

(b) When moisture content of mixed materials was from $10 \%$ to $12 \%$, the physical properties of molding fuel were better.

(c) When molding pressure was from $25 \mathrm{mpa}$ to $30 \mathrm{mpa}$, the physical properties of molding fuel were better.

\section{References}

1. Wang Jiuchen, Dai Lin, Tian Yishui, et al. Analysis of the development status and trends of biomass energy industry in China [J]. Agricultural 
Engineering, 2007 (09): 276-282(In Chinese).

2. Tian Yishui, Zhao Lixin, Sun Liying, et al. Analysis and evaluation on agricultural biomass resources $[\mathrm{J}]$. China Engineering Science, 2011(02):24-28(In Chinese).

3. Wu Chuangzhi, Zhou Zhaoqiu, Yin Xiuli, et al. Current status of biomass energy development in China $[\mathrm{J}]$. Transactions of the Chinese Society for Agricultural Machinery, 2009(01):91-99(In Chinese).

4. Zhou Likun. Research status and prospect to the sludge cleaning technology of oil tank [J]. Gas Storage and Transportation, 2013(03):229-235(In Chinese).

5. Li Jianzhu, Li Xiao'ou, Feng Ruijiang, et al. Oily sluge and the development of oily sluge treatment technologies[J]. Refining Technology and Engineering, 2009(12):1-4(In Chinese).

6. Zhao Dongfeng, Zhao Chaocheng, Lu Shuai. The study on oily sludge coking process $[\mathrm{J}]$. Research of Environmental Sciences, 2000(02):55-57(In Chinese).

7. Lin Weibang, Jiang Weifen, Zheng Gang. The new progress of oily sludge treatment technology[J]. Guangzhou Chemical Industry, 2013(15):14-15(In Chinese). 